\title{
Über dermatologische Weltkongresse und Regionalveranstaltungen
}

About Dermatological World Congresses and Regional Events

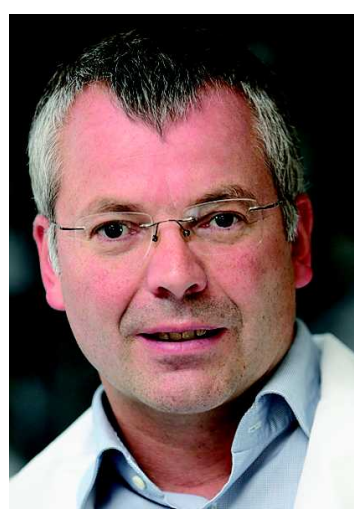

Prof. Dr. Peter von den Driesch
Fast 20000 Teilnehmer aus aller Welt trafen sich vom 30.9. bis zum 5.10.2007 in Buenos Aires zum 21. Weltkongress der Dermatologie. Die Veranstaltung stand unter dem Motte „Globale Dermatologie in einer globalisierten Welt“. Veranstalter war die Internationale Liga der dermatologischen Vereinigungen, der auch die Deutsche Gesellschaft für Dermatologie angehört. Als Teilnehmer auf diesem Kongress erlebte ich das gesamte Spektrum an Eindrücken, die ein solcher gigantischer Kongress zu vermitteln in der Lage ist. Nach einer stimmungsvollen Auftaktveranstaltung mit eindrucksvollen landestypischen Tanzdarstellungen und Begrüßung durch den Vizepräsidenten des Landes Argentinien zeigte sich in den nächsten Tagen ein außerordentlich vielfältiges wissenschaftliches Programm, bei dem der Teilnehmer häufig auch die Qual der Wahl zwischen verschiedenen Parallelveranstaltungen hatte. Hierbei waren in den insgesamt gut besuchten Veranstaltungen sowohl die inhaltliche Qualität der Beiträge als auch deren technische Umsetzung sehr variabel. Der Teilnehmer musste einiges an organisatorischem Geschick mitbringen, um jederzeit im richtigen Gebäude und dann auch im richtigen Saal zu sein. Überwand man diese Hemmschwellen, war es schon eine Freude, den berühmtesten Dermatologen unserer Zeit bei ihren verschiedenen Vorträgen zuhören zu können. Allerdings ergab sich schon aus der enormen Anzahl der Zuhörer, dass eine geregelte Diskussion der Vorträge eher selten zustande kam.

Auch die Industrieausstellung übertraf in ihren Dimensionen fast alles, was der Autor bisher auch auf großen Kongressen erlebt hat. Gewöhnungsbedürftig war aber, dass sich an den Ständen aller namhafter pharmazeutischer Hersteller ungewohnt lange Schlangen bildeten, so dass die Fachgespräche sich auf ein zügiges Aushändigen der gewünschten Muster beschränken mussten. Nimmt man die Strapazen der langen An- und Abreise mit in die Rechnung, stellt sich am Ende doch die Frage, ob der fachliche Gewinn diesen Aufwand wirklich gerechtfertigt hat.

Im Gegensatz hierzu, das sei mir an dieser Stelle gestattet, möchte ich eine Lanze für unsere ge- wohnten und seit Jahrzehnten erfolgreich etablierten Regionalkongresse brechen. Hierzu zähle ich die Veranstaltungen der Norddeutschen Dermatologischen Gesellschaft, der Vereinigung Rheinisch-Westfälischer Dermatologen, der Vereinigung Südwestdeutscher Dermatologen und der Vereinigung Südostdeutscher Dermatologen. Was ist aus meiner Sicht der Vorteil dieser Veranstaltungen, gerade wenn man unter dem Eindruck eines solchen Megakongresses wie in Buenos Aires steht? Diese Veranstaltungen haben eine ausreichende Größe und Bekanntheitsgrad, um die Dermatologen einer bestimmten Region unseres Landes erfolgreich zu versammeln. Aufgrund dieser bestehenden Etablierung im Bewusstsein der Dermatologen sind sie auch ein Anziehungspunkt für die Industrie, mit dem Vorteil, dass der Dermatologe hier auch wirklich in der Regel seinen regional zuständigen Ansprechpartner findet und daher viel gezielter und frei von Sprachbarrieren Gespräche führen kann. Diese positive Bilanz führt sicherlich auch dazu, dass die großen Regionalveranstaltungen ausreichend von der Industrie unterstützt werden.

Das Programm dieser Regionalveranstaltungen ist sehr gut strukturiert, bietet Kurse für Assistenten und weitere spezielle Fortbildungsmöglichkeiten, den Hauptteil des Programmes bilden aber Vorträge der regional verantwortlichen ärztlichen Direktorinnen und Direktoren der dermatologischen Kliniken. Diese Vorträge werden auch stets ohne die Konkurrenz von Parallelveranstaltungen angeboten. Eine Diskussion ist selbstverständlich bei diesen Tagungen. Dies bietet den Dermatologen die Möglichkeit, mit den regional bekanntesten Vertretern ihres Fachs in Kontakt und Austausch zu treten. Die Themen sind von praktischem Interesse und klinischer Relevanz.

Gestatten Sie daher, dass ich an dieser Stelle noch einmal deutlich machen möchte, wie wichtig diese traditionsreichen Veranstaltungen aus meiner Sicht für die Dermatologie sind.

Prof. Dr. Peter von den Driesch
Stuttgart · New York

ISSN 0340-2541

Korrespondenzadresse

Prof. Dr. med.

Peter von den Driesch

Krankenhaus Bad Cannstatt

Klinik für Dermatologie und

Allergologie

Prießnitzweg 249

70374 Stuttgart

PDriesch@kbc-intern.de
Bibliografie

Akt Dermatol 2007; 33; 401 